\title{
Regular Exercise or Food Restriction, Which is Better in the Event of Heart Failure? An Approach to Oxidative Stress and Angiogenesis
}

\author{
${ }^{1}$ Fatemeh Moradi, ${ }^{1}$ Alireza Imani, ${ }^{1}$ Abbas Shakoori, ${ }^{2,3}$ Mohammad Reza Vaez Mahdavi, ${ }^{4}$ Saman \\ Mehrabi, ${ }^{5}$ Mohammad Molazem, ${ }^{1}$ Marjan Aghajani, ${ }^{1}$ Mahdieh Faghihi ${ }^{*}$
}

${ }^{1}$ Physiology Department, Faculty of Medicine, Tehran University of Medical Sciences, Tehran, Iran. ${ }^{2}$ Equity and Health Research Center, Shahed University, Tehran, Iran. ${ }^{3}$ Physiology Department, Shahed University, Tehran, Iran. ${ }^{4}$ Genetic Department, Faculty of Medicine, Tehran University of Medical Sciences, Tehran, Iran. ${ }^{5}$ Radiology and Surgery Department, Faculty of Veterinary Medicine, University of Tehran, Tehran, Iran.

\begin{abstract}
The aim of present study was to investigate whether food restriction combined with exercise training could attenuate the oxidative stress and promote angiogenesis in a rat model of heart failure. 50 male wistar rats weighing 250-300 g were randomly divided into 5 groups including: 1) sham; they were fed ad libitum food, $n=10$. 2) Heart failure group; 3) Exercise group; they run on a treadmill 5 days per week for 4 weeks, $n=10$. 4) Food restricted group; they were fed with $60 \%$ of their daily average food intake, $n=10.5$ ) Food restricted plus exercise group; as well as feeding with $60 \%$ of their daily average food intake for 8 weeks and run on a treadmill 5 days for the 4 next weeks, $n=10$. Subcutaneous injection of isoproterenol $(130 \mathrm{mg} / \mathrm{kg})$ was used to induce experimental heart failure. Echocardiographic parameters were monitored. Plasma levels of malondialdehyde (MDA) and prooxidant/ antioxidant balance (PAB), as oxidative parameters were measured. In continue gene expression of angiogenic factors such as hypoxia inducible factor-1a (HIF-1a), vascular endothelial growth factor A (VEGFA) and endothelial nitric oxide synthase (eNOS) as well as histopathological examination were investigated. Isoproterenol-treated hearts showed lower functional indexes including LVEDd; Left Ventricular End Diastolic dimension ( $\mathrm{p}<0.05)$, FS; Fractional Shortening ( $\mathrm{p}<0.001)$, EF; Ejection Fraction, $(\mathrm{p}<0.001)$. In addition, significant increase in plasma levels of MDA $(\mathrm{p}<0.001)$ and PAB $(\mathrm{p}<0.001)$ were observed. Food restriction and exercise significantly improved all measured parameters. The protective role of food restriction and exercise training on myocardial damage was further confirmed by promoting the gene expression of angiogenic factors $(\mathrm{p}<0.001)$ in left ventricle and reducing the myocardial fibrosis $(\mathrm{p}<0.05)$. Our results suggest that combined food restriction with exercise training is superior to either therapy alone for improving functional indexes, strengthen balance of antioxidative defense system, as well as gene expression of angiogenic factors and decreasing myocardial fibrosis.
\end{abstract}

KEY WORDS: Regular Exercise, Food Restriction, Heart Failure, Oxidative Stress, Angiogenesis.

\section{INTRODUCTION}

Chronic Heart Failure (CHF) is a major health problem and a leading cause of major disability in many societies (1). In chronic heart failure, myocardial necrosis occurs as a result of

*. Corresponding Author:

Mahdieh Faghihi

E-mail: faghihim@tums.ac.ir 
increased myocardial workload, which is caused by an imbalance in the coronary blood supply and myocardial demand. The patient may experience sever disability, or even die (2).

The key pathophysiological maladaptive process that leads to $\mathrm{CHF}$ is cardiac remodeling, a term referring to intolerable cardiac stress at the molecular, cellular, tissue, and organ levels (3). Left ventricular (LV) remodeling and enlargement is a dynamic response of the heart to the extra workload and heart damage changes and has a major role in the development of heart failure. An increased generation of reactive oxygen species in the heart can directly lead to an increased risk of necrosis and rate of apoptosis in cardiomyocytes and, subsequently, causes cardiac remodeling and dysfunction. Therapeutic outcomes, with an emphasis on strengthening the endogenous antioxidant enzymes activity, or suppressing the free radicals generation, may limit the ischemic injures, infarct size, decrease cell death rate and, consequently, attenuate myocardial dysfunctions (4).

Ischemia-induced angiogenesis takes place as a compensatory mechanism to provide a blood supply for ischemic tissues and preserves the blood flow in the ischemic heart. Angiogenesis is defined as the formation of new blood vessels from the preexisting ones. Recently, hypoxia inducible factor-1a (HIF-1a) and vascular endothelial growth factor A (VEGFA) signaling pathways have been demonstrated to play an important role in the neovascularization response against pathological conditions, such as ischemia and pressure-overload (5).

Studies have indicated the protective effect of intermittent fasting, or the calorie restriction against cardiac-specific risk factors $(6,7)$, whereas the effect of food restriction on angiogenesis has remained unclear. An earlier study by Ahmet et al. (8) demonstrated the reduction of apoptosis in rats that had been fasted on alternative days before ischemia. However, it is not well-known if the same beneficial effect can be obtained under chronic food restriction, exercise training and established cardiac pathologies, such as CHF. Exercise is recommended to improve peripheral alterations induced with $\mathrm{CHF}$, but little research has explored the effects of exercise, with or without food restriction, as an adjunct therapy for CHF.

Calorie restriction (CR) or food restriction (FR) has been shown to improve a variety of physiological parameters over time, especially reduced metabolic rate and oxidative damage, resulting in a decrease in the incidence and severity of cardiovascular diseases (9) and the promotion of angiogenesis (10-12). Some recent studies have shown that both lifelong exercise and lifelong caloric restriction extend the mean and maximal life span, respectively, in a variety of species (13-15). However, little is known regarding the relationship between either of these interventions and the promotion of gene expression or amelioration of oxidative stress effects in heart failure. Therefore, this experiment was designed to investigate the effect of food restriction and promotion of exercise, as well as the application of treadmill running during food restriction, on plasma levels of malondialdehyde (MDA), prooxidant/antioxidant balance (PAB), angiogenic factors and dynamic indexes of the heart in wistar rats.

\section{MATERIALS AND METHODS}

Animals. The experimental protocol was approved by the institutional care and the committee of the Tehran University of Medical Sciences (Tehran, Iran). In this experiment, 50 male wistar rats (weighing 250-300 $\mathrm{g}$ at the beginning of the study) were housed in controlled environment conditions $\left(22 \pm 2{ }^{\circ} \mathrm{C}\right.$; light-dark cycle 7 am-7 pm). The rats were allowed access to water and standard laboratory food ad libitum. The animals were allowed to adapt to their surroundings for at least 6 days before the experiments began. The rats were randomly divided into 5 groups, as follows. Group 1: Sham rats $(n=10$, continued to be fed ad libitum and received 2 subcutaneous injections of saline); Group 2: Isoproterenol (ISO) treated rats $(\mathrm{n}=10$, received 2 subcutaneous injections of ISO $(130 \mathrm{mg} / \mathrm{kg})$ ); Group 3: FR+ISO rats ( $\mathrm{n}=10$, were fed first with $60 \%$ of their average food intake (food restricted) during the 8 weeks, then received 2 subcutaneous injections of ISO); Group 4: $\mathrm{E}+\mathrm{ISO}$ rats $(\mathrm{n}=10$, were fed ad libitum, then in later run-in period of 4 weeks on treadmill as 
exercise activity, finally received 2 subcutaneous injections of isoproterenol); and Group 5: $\mathrm{FR}+\mathrm{E}+\mathrm{ISO}$ rats $(\mathrm{n}=10$, were fed first with $60 \%$ of their average food intake during the 8 weeks, then run-in period of 4 weeks on a treadmill from week 4 of food restriction, finally received 2 subcutaneous injections of isoproterenol).

Exercise Training Protocol. The rats in the training intervention groups ran on a treadmill (4-lane animal treadmill; IITC Life Science Inc., USA) one session daily, for 5 days a week for 4 weeks. Initially, the rats were acclimatized to run for $10-15 \mathrm{~min}$ at $5-7 \mathrm{~m} / \mathrm{min}, 0^{\circ}$ slope for 2 days before the formal treadmill training sessions. Initially, electrical shocks $(1.0 \mathrm{~mA})$ were used to force animals to run forward. Subsequently, they ran without electrical stimulation. After an adaptive running session, the rats started formal training. The rats in the training groups were scheduled to run on the treadmill for all 4 weeks. The formal treadmill training was started with a speed of $18 \mathrm{~m} / \mathrm{min}$ for $35 \mathrm{~min}$ and a $0^{\circ}$ slope for 5 days per week in the first week. The animals ran for $40 \mathrm{~min}$ at $18 \mathrm{~m} / \mathrm{min}$ with a $5^{\circ}$ slope, 45 $\mathrm{min}$ at $18 \mathrm{~m} / \mathrm{min}$ with a $10^{\circ}$ slope and $50 \mathrm{~min}$ at $18 \mathrm{~m} / \mathrm{min}$ with a $15^{\circ}$ slope, respectively, in the second, third and fourth weeks of training. Sedentary animals were placed on a stationary treadmill daily and were given electrical stimulation in a manner identical to that used for the exercise group (16).

Rat Model of Heart Failure. To induce experimental heart failure, after 8 weeks of study, ISO was dissolved in normal saline and injected subcutaneously to rats $(130 \mathrm{mg} / \mathrm{kg})$ daily for two consecutive days (17).

Blood Sampling. Blood samples were collected from retro-orbital plexus at days 1, 3 and 5 after ISO injections for biochemical analysis (MDA and PAB). The samples were centrifuged at $5000 \mathrm{rpm}$, and $4^{\circ} \mathrm{C}$, for $15 \mathrm{~min}$. The serum was then removed and stored at $-70^{\circ} \mathrm{C}$ until biochemical analysis.

Echocardiographic Assessments. On the $30^{\text {th }}$ day after ISO injections were given, transthoracic echocardiography was performed while the rats were maintained under pentobarbital sodium anesthesia $(60 \mathrm{mg} / \mathrm{kg})$. Two-dimensional and M-mode images were obtained using a $10 \mathrm{MHz}$ transducer connected to an ultrasonic echocardiographic system (Clear
Canvas by Synaptive Medical Toronto, Canada). Systolic and diastolic dimensions of the LV, fractional shortening (FS), left-ventricular post wall thickness (LVPWT) and ejection fraction (EF) were obtained from the M-mode view. All variables were measured in triplicate and averaged. The investigator who performed the echocardiograms was blinded to the treatment group.

Tissue Preparation and Histological Analysis. Once the physiological measurements were completed on the $30^{\text {th }}$ day of ISO injections, the rats were heavily anesthetized with ketamine $(100 \mathrm{mg} / \mathrm{kg})$ and xylazin (25 $\mathrm{mg} / \mathrm{kg}$ ) and sacrificed. The myocardial samples of some animals from each group $(n=5)$ were immediately removed, rinsed in PBS, frozen in liquid nitrogen and stored at $-80^{\circ} \mathrm{C}$ for evaluation of gene expression. The hearts of others were removed, weighed, and cut into transverse slices at the mid-papillary muscle level. They were then fixed in $10 \%$ buffered formalin, embedded in paraffin, cut into 4- $\mu \mathrm{m}$ thick sections and stained with Masson's trichrome. Measurements were derived from cross-sections at approximately half of the distance between the apex and base. Images were acquired by using a binocular microscope (Optiphot, Nikon, Melville, NY) with a camera and image processor (DKC5000, Sony, New York, NY).

Assessment of Oxidative Stress by TBARS Estimation. To evaluate the oxidative indicator, serum levels of MDA (marker of lipid peroxidation) were assessed by reaction with thiobarbituric acid (TBA). MDA, or MDA-like substances, and TBA react to produce a pink pigment with maximum absorption at $532 \mathrm{~nm}$. In brief, $50 \mu \mathrm{l}$ of serum, $1 \mathrm{ml}$ of TBA: $1 \mathrm{ml}$ of trichloroaceticacid (TCA) [0.75\% TBA: $30 \%$ TCA] were mixed and placed in a boiling water bath for $90 \mathrm{~min}$, cooled and centrifuged at $4{ }^{\circ} \mathrm{C}$ for $10 \mathrm{~min}$ at $3000 \mathrm{rpm}$. The absorbance of the supernatant was measured against a reference blank at $532 \mathrm{~nm}$ by spectrophotometer. 1,1,3,3Tetramethoxypropane (Sigma Chemicals, USA) was used as a standard. The results were expressed as $\mathrm{nmol} / \mathrm{ml}$ of serum.

Measurement of PAB. Serum levels of PAB (markers of oxidative stress) were measured by a colorimetric method, with specific PAB kits 
(Pars Azmoon, Tehran, Iran), using an auto analyzer (Roche Hitachi Modular DP Systems; Mannheim, Germany) according to the manufacturer's instructions. The results were expressed as a HK unit.

Quantitative Real-time PCR (qPCR) Assays. Total RNA in the LV samples was isolated with Trizol reagent (Invitrogen, Shanghai, China). For cDNA synthesis, $1.0 \mathrm{mg}$ RNA was used and the reactions were carried out using a reverse transcribed system (Takara, Japan). RT-PCR was performed in a Genemate thermal cycler (Jinge Instr, Hangzhou, China). Quantification of gene expression was performed using the Rotor-Gene 6000 (Qiagene). The value for each sample was an average of three independent PCR measurements.

Quantitative real-time PCR (qPCR) was performed using a SYBR Green Master Mix (Takara Bio, Inc.) The cycle threshold (CT) values were automatically determined in triplicate and averaged. All real-time PCR sample reactions were normalized to the HPRT gene as the housekeeping expression. A standard curve was run with the dilution series of the amplified fragment, allowing for mRNA copy number calculation. The relative expression of VEGFA, HIF-1a and eNOS were calculated using the $2^{-\Delta \Delta C T}$ method (18). The specific primer sequences are listed in Table3.

Mortality and Survival. There was no mortality in the sham group. A total of four animals died during protocols; three animals in the ISO group (3/10) and one animal in the
E+ISO group (1/10) respectively, following ISO administration.

Data Analysis. All data were expressed as mean \pm SEM and, for statistical analyses of data, we used a repeated measure test for the biochemical analysis (MDA and PAB) at days 1, 3 and 5 after ISO injections. A one-way ANOVA with post-test of Tukey was used for other parameter analyses. $\mathrm{p}<0.05$ was accepted as statistically significant. Statistical analyses of data were performed using SPSS statistical software (version 16). The changes in fold of gene expressions were calculated by using REST software version 2009.

\section{RESULTS}

Body and Heart Weights. Isoproterenol induced changes in the heart weight. On the $30^{\text {th }}$ day after ISO administration $(130 \mathrm{mg} / \mathrm{kg}$ daily for 2 consecutive days) in the ISO group, a oneway ANOVA test with post-test of Tukey showed that the heart weight and heart/body weight ratios were increased compared with those of the age-matched sham group injected with saline $(\mathrm{P}<0.001$; Table 1$)$. The application of food restriction, in conjunction with exercise, significantly decreased the heart weight and heart/body weight ratio in FR+E+ISO rats compared with ISO rats $(\mathrm{P}<0.05)$. In body weight, a significant decrease in all foodrestricted animals was also shown in comparison with the sham ad libitum-fed and ISO group $(\mathrm{P}<0.001$, Table 1$)$.

Table 1. Body Weight, Heart Weight, and Heart Weight/Body Weight Ratio

\begin{tabular}{cccccc}
\hline & Sham & ISO & FR+ISO & E+ISO & FR+E+ISO \\
\hline Body weight $(\mathbf{g})$ & $257.3 \pm 1.6$ & $258.7 \pm 1.5$ & $216.3 \pm 5.3^{*} *^{\$}$ & $248.7 \pm 2.4$ & $235.4 \pm 3.7 * *^{\$}$ \\
Heart weight $(\mathbf{m g})$ & $1280 \pm 0.5$ & $1600 \pm 0.35 * *$ & $1252 \pm 0.5^{\$}$ & $1500 \pm 0.03 * \$$ & $1130 \pm 0.04^{\$}$ \\
Heart/Body weight $\mathbf{( m g / g )}$ & $4.9 \pm 0.16$ & $6.2 \pm 0.4 * *$ & $5.8 \pm 0.22^{*}$ & $6.04 \pm 0.16 *$ & $4.8 \pm 0.24$ \\
\hline
\end{tabular}

Echocardiography Findings. Echocardiographic data obtained on the 30th day after ISO injections showed that the one-way ANOVA with post-test of Tukey showed changes in the functional indexes in the rats' hearts in favor of heart failure (data are summarized in Table 2, Fig.1). FS and EF significantly decreased and LVEDd was significantly increased in the ISO group as opposed to the other groups $(\mathrm{P}<0.05)$. LVEDd returned to the level of the sham rats only in the $\mathrm{FR}+\mathrm{E}+\mathrm{ISO}$ rats $(\mathrm{P}<0.05)$. Decreased FS and EF was shown in the ISO group, but reversed with food restriction and treadmill exercise in $\mathrm{FR}+\mathrm{ISO}, \mathrm{E}+\mathrm{ISO}$ and $\mathrm{FR}+\mathrm{E}+\mathrm{ISO}(\mathrm{P}<0.05)$. 


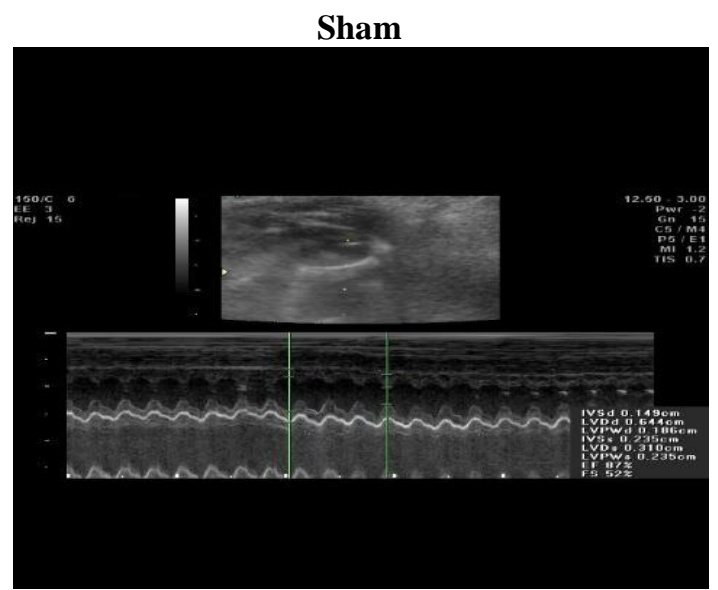

FR+ISO

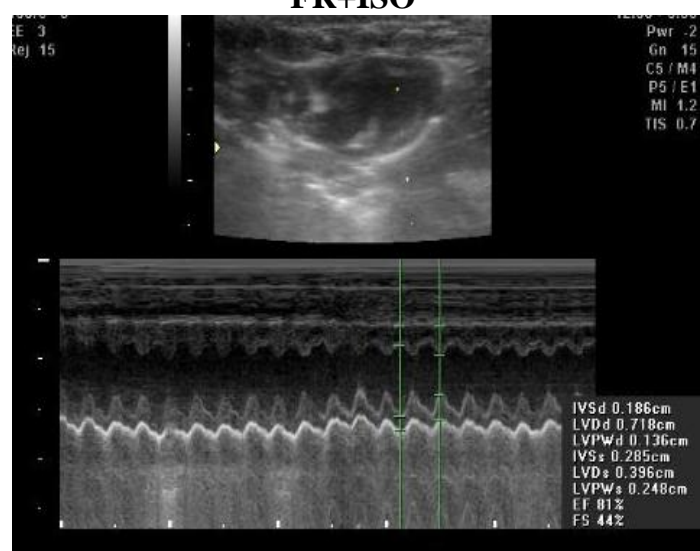

ISO

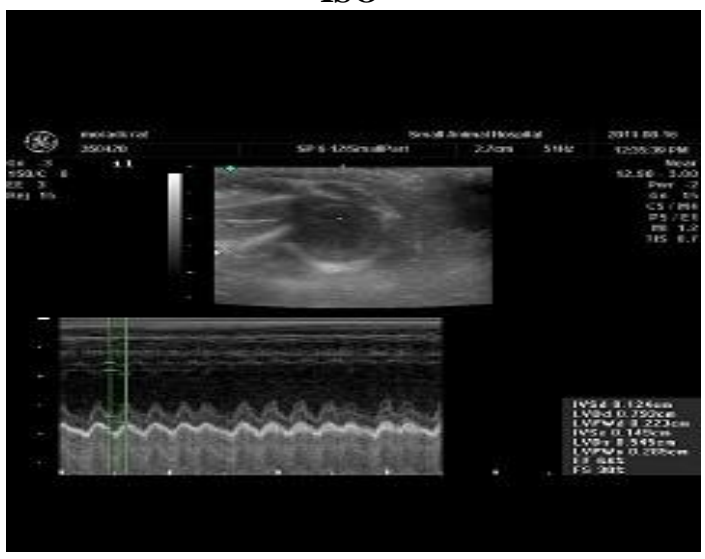

E+ISO

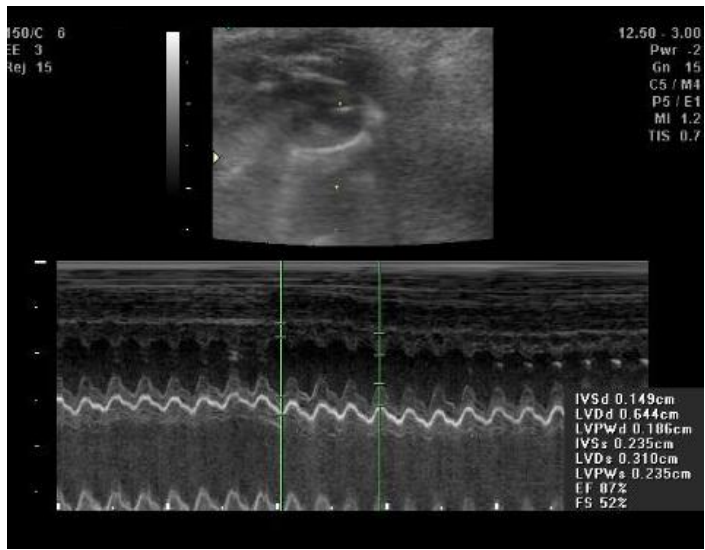

FR+E+ISO

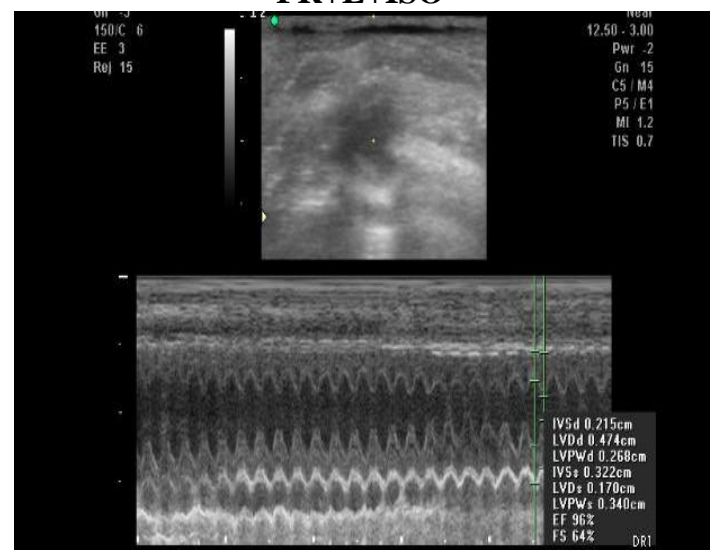

Fig.1. Photographs of echocardiography on 30th day after injections of isoproterenol in experimental groups. ISO; Isoproterenol, FR; Food Restriction, E; Exercise.

Table 2. Effects of food restriction and exercise on cardiac remodeling and function evaluated by echocardiography on $30^{\text {th }}$ day after injections of isoproterenol.

\begin{tabular}{cccccc}
\hline & Sham & ISO & FR+ISO & E+ISO & FR+E+ISO \\
\hline LVESd (cm) & $0.15 \pm 0.009$ & $0.17 \pm 0.005$ & $0.16 \pm 0.006$ & $0.14 \pm 0.14$ & $0.18 \pm 0.16$ \\
\hline LVEDd (cm) & $0.67 \pm 0.23$ & $0.73 \pm 0.02^{*}$ & $0.73 \pm 0.01^{*}$ & $0.72 \pm 0.03^{*}$ & $0.66 \pm 0.03^{\$ \&}$ \\
\hline LVPWT (cm) & $0.17 \pm 0.008$ & $0.20 \pm 0.009$ & $0.20 \pm 0.02$ & $0.19 \pm 0.22$ & $0.19 \pm 0.01$ \\
\hline EF (\%) & $77.8 \pm 2.6$ & $62 \pm 2.1^{* *}$ & $76 \pm 2.1^{\$}$ & $86 \pm 1.2^{\$}$ & $72+2.06^{\$}$ \\
\hline FS (\%) & $63.8 \pm 4.1$ & $34 \pm 1.7^{* *}$ & $61.1 \pm 5.5^{\$}$ & $62.4 \pm 4.2^{\$}$ & $66.07 \pm 1.9^{\$}$ \\
\hline
\end{tabular}


MDA levels. As shown in Figure 2, the oneway ANOVA with post-test of Tukey showed that induction of heart failure with ISO injections significantly increased MDA levels at days 1,3 and 5 in the ISO group in comparison with the sham group $(\mathrm{P}<0.05)$. Not only did food restriction and exercise training decrease MDA levels in comparison with the ISO group at days 1,3 and $5(\mathrm{P}<0.05)$, but also the MDA levels returned to sham levels on day 5. Moreover, combined food restriction with exercise training was superior to either therapy alone for decreasing MDA levels at day 5 after ISO injections. MDA levels at days 3 and 5 were significantly decreased in all ISO-injected rats in comparison with day $1 \quad(\mathrm{P}<0.05)$. A repeated measure analysis showed that MDA levels were significantly decreased at days 3 and 5 in comparison with day $1(\mathrm{P}<0.05)$ in all ISOinjected rats.

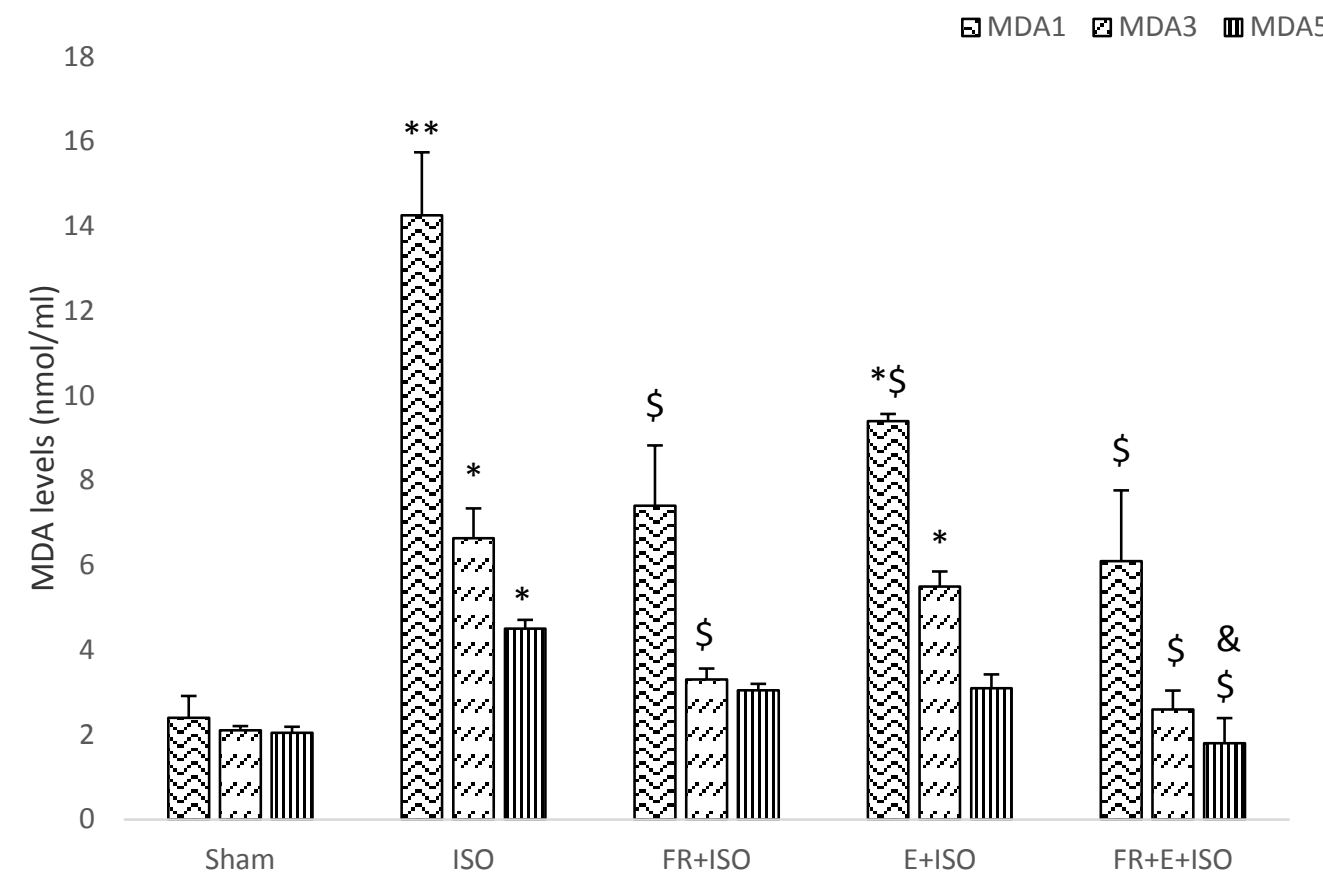

Fig.2. Serum levels of malondialdehyde (MDA) at days 1, 3 and 5 after injections of isoproterenol $(n=6)$. ISO; Isoproterenol; FR, Food Restriction; E; Exercise, data are presented as means \pm SEM. $* \mathrm{P}<0.05$ vs. sham, $* * \mathrm{P}<0.001$ vs. sham, $\$ \mathrm{P}<0.05$ vs. ISO, \& $\mathrm{P}<0.05$ vs. FR+ISO and vs. E+ISO.

Prooxidant Antioxidant Balance (PAB). As shown in Figure 3, in all the ISO-treated rats, the one-way ANOVA with post-test of Tukey showed a significant increase in serum total PAB levels 1 day after ISO injections compared to the sham rats $(\mathrm{P}<0.001)$. PAB levels in $\mathrm{FR}+\mathrm{ISO}$, $\mathrm{E}+\mathrm{ISO}$ and $\mathrm{FR}+\mathrm{E}+\mathrm{ISO}$ rats significantly decreased on day 5 of the ISO injections in comparison with that seen in ISO rats $(\mathrm{P}<0.05)$.
Pretreatment with food restriction, followed by exercise training, in $\mathrm{FR}+\mathrm{E}+\mathrm{ISO}$ rats could be more effective than FR or $\mathrm{E}$ alone in decreasing the PAB level near to sham level on day 5 of the ISO injections $(\mathrm{P}<0.05)$. Repeated measure analysis showed that the PAB levels at day 5 had significantly decreased in all ISO-injected rats in comparison with day $1(\mathrm{P}<0.05)$. 


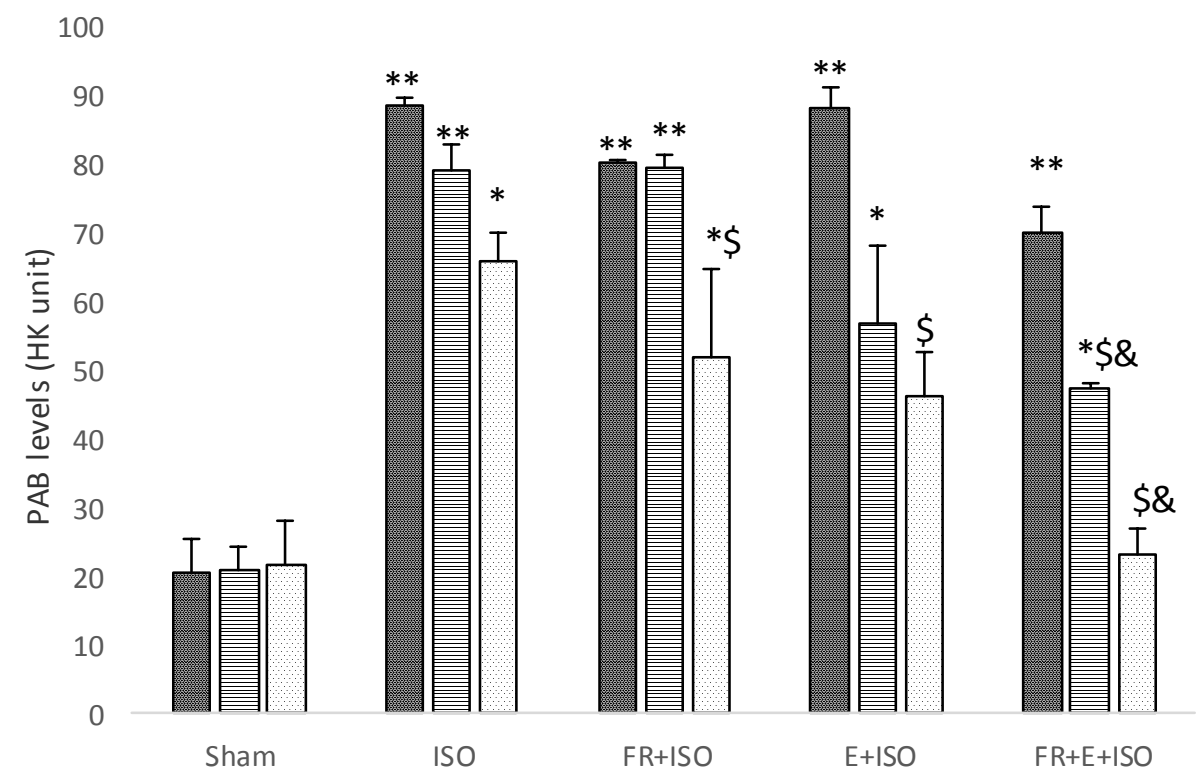

Fig.3. Serum levels of Prooxidant/ Antioxidant Balance (PAB) at days 1, 3 and 5 after injections of isoproterenol ( $\mathrm{n}=6)$. ISO; Isoproterenol; FR, Food Restriction; E; Exercise, data are presented as means \pm SEM. ${ }^{*} \mathrm{P}<0.05$ vs. sham, $* * \mathrm{P}<0.001$ vs. sham, ${ }^{\$} \mathrm{P}<0.05$ vs. ISO, ${ }^{\&} \mathrm{P}<0.05$ vs. FR+ISO and vs. E+ISO.

Quantitative real-time RT-PCR. We investigated the potential mechanisms for food restriction and exercise action on the mRNA expression of growth factors involved in myocardial angiogenesis. A total of 25 hearts were used for Real-Time PCR Analysis of mRNA expression of VEGFA, HIF-1a and eNOS. Fig. 4 shows that pretreatment with food restriction and regular exercise significantly increased HIF-1a and eNOS $(\mathrm{P}<0.05)$ when compared to the sham and ISO animals. Gene expressions of VEGFA in all food-restricted animals were increased in comparison with the sham and ISO animals $(\mathrm{P}<0.05)$. The expression of all these angiogenic factors in FR+E+ISO animals was significantly increased when compared with the sham, ISO, E+ISO and FR+ISO groups $(\mathrm{P}<0.05)$. Our data analysis showed no significant differences in the mRNA expression between ISO and sham animals.

Histopathological Finding. Masson's trichrome staining of LV heart tissue sections from sham animals (Fig.5) showed no obvious fibrosis and pathological abnormalities and had intact cardiac morphology. Quantitative histologic analysis of cross-sections from the LV in ISO-treated rats demonstrated a marked increase in the collagen network of the myocardium. The mean of collagen deposition of the LV endocardium was significantly higher in ISO rats $(28.8 \pm 1.5)$ compared with that seen in the sham rats $(5.5 \pm 1.7)(\mathrm{P}<0.001)$. Also, the mean of collagen deposition significantly decreased in FR+ISO (14.5 \pm 1.4$), \quad$ E+ISO $(13.1 \pm 1.1)$ and $\mathrm{FR}+\mathrm{E}+\mathrm{ISO} \quad(7.7 \pm 1.3)$ rats compared with that seen in the ISO rats $(\mathrm{P}<0.05$, Fig.7). However, mild myocardial degeneration and collagen deposition was observed in the FR+ISO and E+ISO-induced group when compared to the sham group. Food restriction associated with exercise training could minimize the pathological changes and had more LV tissue protection from fibrosis induced with ISO injections. 


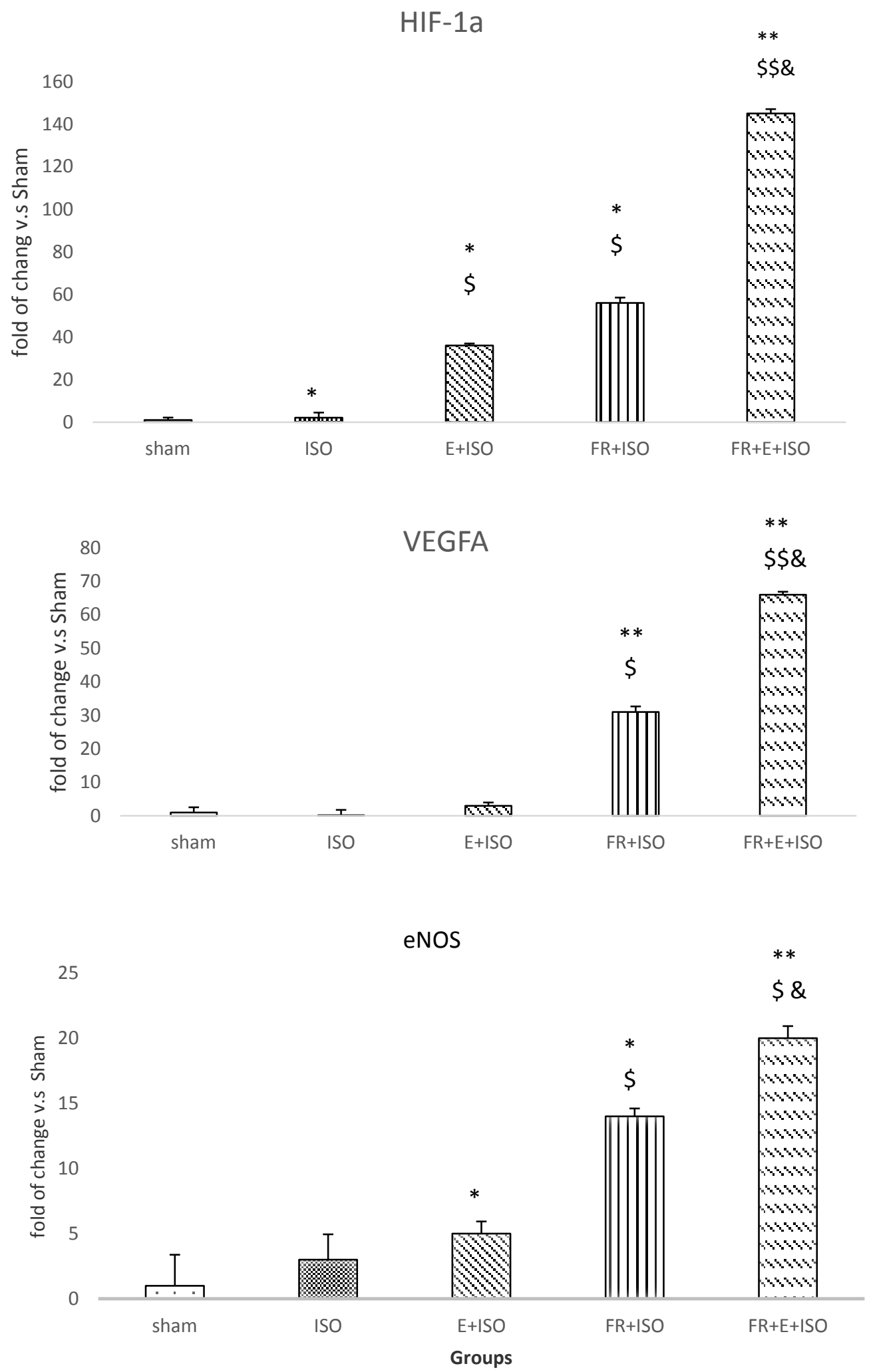

Fig.4. Effects of food restriction and exercise on HIF-1a, VEGFA and eNOS mRNA expression in LV ( $\mathrm{n}=5$ ). ISO; Isoproterenol; FR, Food Restriction; E; Exercise, data are presented as means $\pm \mathrm{SEM}$. $* \mathrm{P}<0.05$ vs. sham, $* * \mathrm{P}<$ 0.001 vs. sham, ${ }^{\$} \mathrm{P}<0.05$ vs. ISO, ${ }^{\&} \mathrm{P}<0.05$ vs. FR+ISO and vs. E+ISO 
Table 3. The sequence of primers used in Real-Time PCR

\begin{tabular}{clc}
\hline Gene name & \multicolumn{1}{c}{ Primer sequence PCR } & product size \\
\hline \multirow{2}{*}{ HIF-1a } & $\begin{array}{l}\text { Forward: ACAGAAATGGCCCAGTGAGAAA } \\
\text { Reverse:TGAATGTGGCCTGTGCAGTG }\end{array}$ & 148 \\
\hline \multirow{2}{*}{ VEGFA } & $\begin{array}{l}\text { Forward: CAAACCTCACCAAAGCCAGC } \\
\text { Reverse: GGTCTTTCCGGTGAGAGGTC }\end{array}$ & 181 \\
\hline \multirow{2}{*}{ eNOS } & $\begin{array}{l}\text { Forward: GGTGACCAGCACATTTGGCA } \\
\text { Reverse: GGGCCCGACATTTCCATCAG }\end{array}$ & \multirow{2}{*}{457} \\
\hline \multirow{2}{*}{ HPRT } & $\begin{array}{l}\text { Forward: CTCATGGACTGATTATGGACAGGAC } \\
\text { Reverse: GCAGGTCAGCAAAGAACTTATAGCC }\end{array}$ & \multirow{2}{*}{123} \\
\hline
\end{tabular}

A

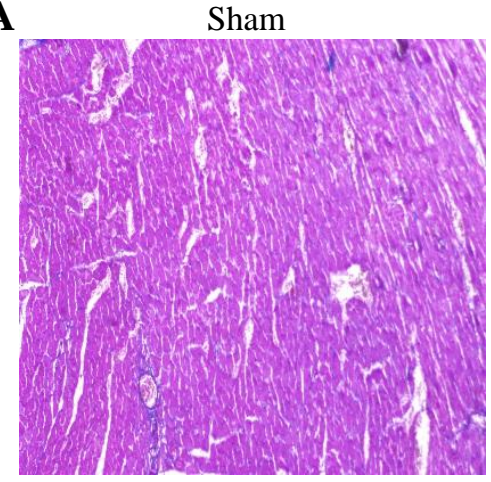

E+ISO

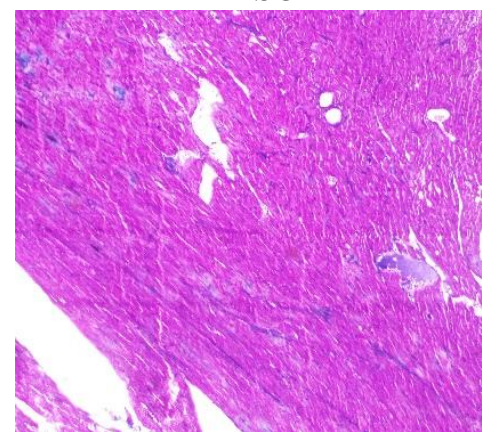

35

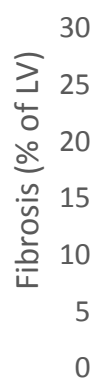

B

sham

$\mathrm{FR}+\mathrm{E}+\mathrm{ISO}$
ISO
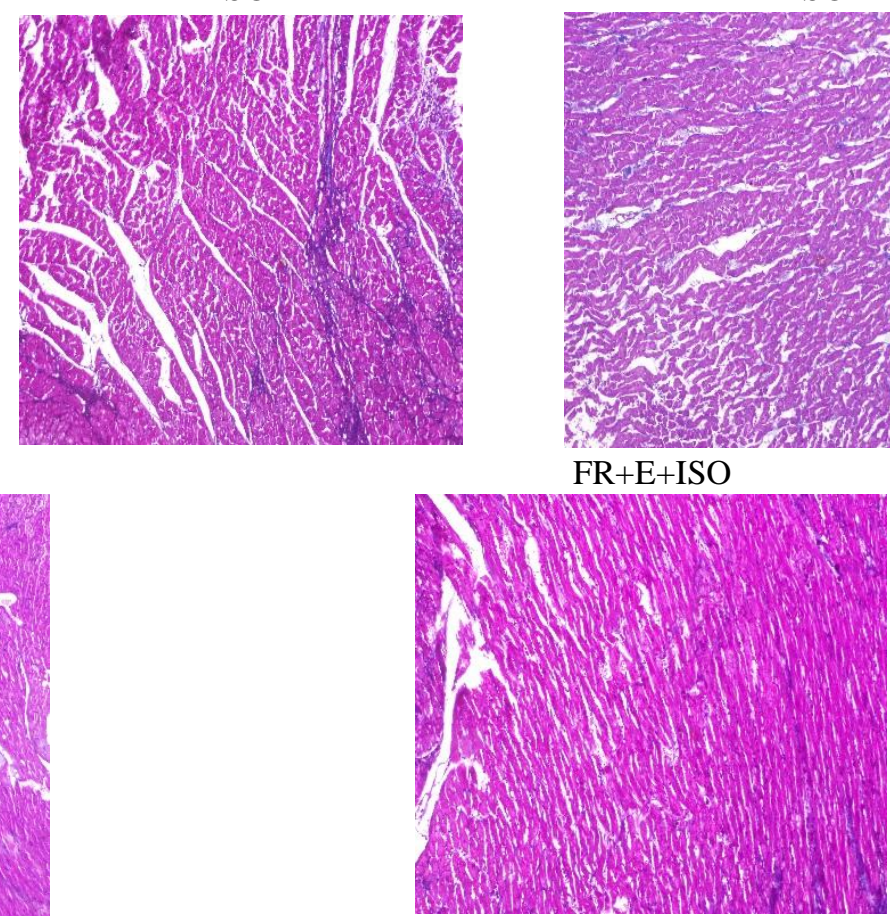

FR+ISO

$\mathrm{FR}+\mathrm{E}+\mathrm{ISO}$
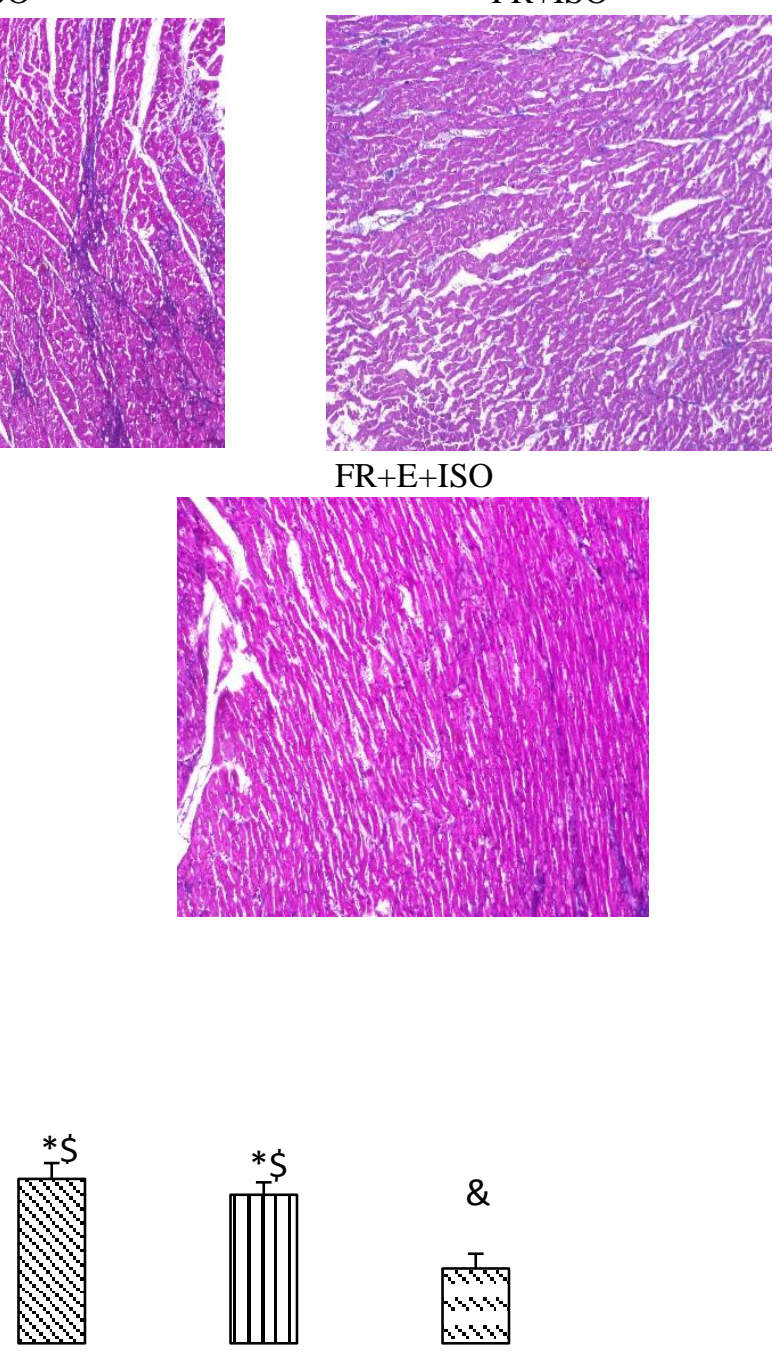

\&

FR+ISO

ISO

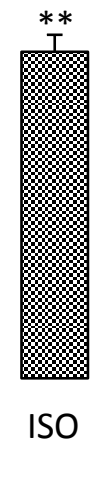

Fig.5. Histological evaluation of food restriction and exercise on the isoproterenol induced heart failure (Masson's trichrome stain). A: Ventricular transverse slices, B: Quantification of interstitial fibrosis $(n=4)$. ISO; Isoproterenol; FR, Food Restriction; E; Exercise. 


\section{DISCUSSION}

It is known that synthetic $\beta$-adrenoceptor agonist, such as isoproterenol, causes myocardial infarction in rats, through physiological imbalance between the production of free radicals and antioxidant defense system in the development of cardiac remodeling and CHF. The results of the majority of studies suggest that strengthening the antioxidant defenses may be one of the strongest proposed therapeutic candidates against cardiac remodeling (17).

Therefore, in our study, the noninvasive rat model of heart failure was used to assess the efficacy of food restriction, with or without exercise training, on protecting the heart against oxidative damage, the effects of angiogenic factors, and focused on the correlation between functional, biochemical, genetical and histopathological changes. Our results showed that both food restriction and exercise training in animals are highly effective in improving the oxidative state, echocardiographic indexes, angiogenic factors and histological parameters. Moreover, food restriction combined with exercise training is more efficient at reducing the levels of MDA and PAB after isoproterenolinduced myocardial injury. Therefore, the present results highlighted the ameliorating effects of the conjunction of food restriction and regular exercise upon isoproterenol-induced heart failure involving the modulation of oxidative stress mediators and up-regulation of the pro-angiogenic factors, HIF-1a, VEGFA and eNOS.

Ventricular enlargement and hypertrophy, reduced ejection fraction and fractional shortening have been shown to be important predictors of prognosis in both myocardial infarction and heart failure patients (3). In our study, echocardiographic evaluation after 30 days of ISO administration in FR+ E+ISO rats showed lower LVEDd than those of ISO, FR+ISO and E+ISO animals. These rats had decreased heart weight and heart weight to body weight ratio. One explanation for the smaller heart in these rats is a retardation of growth and increased resistance to remodeling and hypertrophy of the heart prior to ISO injections in young animals experiencing a reduction of food intake, followed by exercise training. However, other ISO-treated rats showed higher heart/body weight ratio, lower left-ventricular ejection fraction, fractional shortening and higher left-ventricular end diastolic dimension.

The results of the majority of the studies suggest that antioxidants may be one of the strongest proposed therapeutic candidates against cardiac remodeling (4). A more direct relationship has been established between the markers of oxidative stress and indexes of functional capacity (4). During the heart failure process, the cardiomyocytes are exposed to a continuous hypoxic state, resulting in the formation of free radical molecules, which induce a variety of cellular destructions $(4,19)$. In our study, the FR+ISO and E+ISO groups showed a reduced level of oxidative stress (supplementary Figs. 2, 3 and 5) when compared to the ISO group with normal food intake. In addition, the FR+E+ISO rats showed more preservation, thus confirming the synergic effect of food restriction and regular exercise. Even though the exact mechanism behind the reduced oxidative stress is not known, the possible mechanisms could be due to the reduced energy intake during the food restriction regimen and activation of antioxidative defenses during the regular exercise, which would reduce the formation of oxidative stress markers in mitochondria and, hence, reduce oxidative damage to the cells (20). The other possible reason could be that the mild stress in the form of food restriction and exercise, before cardiac ischemia and failure, conditions the myocardium against the formation of free radicals at a later date. This is similar to the protective effects of ischemic preconditioning, where mild ischemia before the prolonged ischemia can protect the myocardium from severe ischemic damage (21, 22). Calorie restriction (CR) or food restriction (FR) must be considered separately from food starvation and defined as a reduction in calorie or food intake below the usual ad libitum intake without malnutrition. In the past, experimental studies indicated that, when maintained on an intermittent fasting diet, the mice exhibited increased resistance to many different types of stress (23).

So, both physical activity and food restriction have shown to preserve cardiac function in animals. It can be postulated that their actions may be synergistic. Although the beneficial 
effects of food restriction and exercise training on the survival rate may not be additive $(24,25)$, a combination of long-term caloric restriction and exercise training may have a synergistic effect on the cardiac function. As we have shown, there was an improvement in functional parameters such as a decrease in LVEDd, and an increase in FS and EF after 8 weeks of diet intervention and 4 weeks of exercise. Therefore, our data are currently available regarding the cardioprotection effects of food restriction, combined with exercise training, on restoring the cardiac function in the event of heart failure, partly through the normalization of the levels of antioxidant enzymes.

In addition, it is found that the increased levels of oxidative stress metabolites, both in plasma and pericardial fluid, had a negative correlation with the ventricular function, and spatially LV diastolic dimensions (26). Therefore, plasma levels of MDA may be considered as a reliable marker for the estimation of oxidative stress severity in heart pathologies (19). The results presented in this study indicate that food restriction and treadmill exercise preconditioning could decrease isoproterenol-induced MDA elevation. More importantly, we showed that the action of food restriction and exercise training on oxidative stress in the event of heart failure is additive and, therefore, oxidative balance is completely restored after 5 days. It was quite possible that the antioxidative effects of food restriction and exercise training were effectively neutralized and/or scavenged the free radicals induced by isoproterenol.

It is well-established that angiogenesis plays a critical role in various pathological settings, including tumor growth, wound repair and MI, and may promote cardiac growth and maintain contractile function (17). Several angiogenic factors, including vascular endothelial growth factor (VEGF), hypoxia-inducible factor-1a (HIF-1a), eNOS and others are involved in this process $(5,17)$.

In ischemic conditions, hypoxia-induced angiogenesis takes place in tissues due to the increase in the gene expression of some important growth factors, such as VEGF (17). Also, nitric oxide (NO) has been demonstrated to be an important molecule in the cardiovascular system and is thought to be critical in signal transduction for inducing angiogenesis through the ischemic myocardium. NO is generated by the nitric oxide synthase (NOS) enzyme. Endothelial nitric oxide synthase (eNOS) isoform has been indicated to play an important role in reducing the infarct size during myocardial infarction (27). It has been reported that overexpression of eNOS and VEGF takes place in tumor angiogenesis and metastasis, so eNOS-derived NO is important to promote the survival of ischemic tissue by stimulating angiogenesis (26).

Several experimental studies have proved the improved survival rate with overexpression of angiogenic factors such as VEGF and HIF-1a (5, 17). In our study, food restriction exhibited a significant increase in the expression of VEGFA after HF induction and this protective mechanism had more promotion in $\mathrm{FR}+\mathrm{E}+\mathrm{ISO}$. The other master transcription factor HIF-1- $\alpha$ and eNOS was also significantly elevated in animals that underwent food restriction and exercise conditions compared to those of ISO rats, which were normally fed without physical training. The expression of this factor was also greater in $\mathrm{FR}+\mathrm{E}+\mathrm{ISO}$ compared to the other animals (Fig 6).

Consistent with these results, not only did our data demonstrate that food restriction with exercise attenuated an oxidative state with decreasing MDA and PAB levels, together with gene expression of angiogenic factors, but it led to improved cardiac dysfunction and survival 30 days after $\mathrm{CHF}$ induction.

It has been demonstrated that an increase in the oxidative stress causes cardiac hypertrophy and fibrosis (28). In Masson's trichrome staining of the left ventricular, we showed that ISO also induced an increase in focal necrosis, myocardial degeneration, loss of myofibrillar alignment, severe cytoplasmic vacuolization and the collecting of collagen fibers spatially around the small capillaries. Food restriction and treadmill training followed by ISO dramatically reduced the ISO-induced severe vacuolization, necrosis and the collecting of collagen fibers when compared to ISO-treated rats. The combination of food restriction with exercise training offered an additional benefit compared to either factor 
performed alone in terms of reducing myocardial fibrosis.

The effect of food restriction should be distinguished from that of malnutrition and starvation $(18,20,29)$. In our study, similar to previous experiments in which food intake was restricted to $60-70 \%$ of ad libitum, there was no evidence of malnutrition in animals treated with the standard regimens of food restriction (1012), but a significant decrease in body weight of all food-restricted rats compared to the sham rats took place. It is conceived that the most considerable mechanisms that may be responsible for the beneficial health and physiological effects of CR are usually mediated through attenuation of the oxidative stress (decreased oxidative damage to proteins, lipids and DNA), and increased resistance to various types of stress-mediated oxidative damage, such as metabolic stress (30). In the present study, no rats died during the 8 -week food restriction period and there was no difference in their behavior between ad libitum and food restriction (data not shown). The cardioprotective effects of food restriction and exercise have been extensively studied; however, few clinical and experimental studies have examined the relationships between the conjunction of these situations and cardiovascular disease. This study showed that exercise and food restriction greatly reduced the pathophysiological modifications induced by oxidation and significantly improved echocardiographic indexes, such as fractional shortening and ejection of fraction in rats, but how they exert their anti-oxidation action is not clear.

In summary, our results strongly suggest that food restriction with exercise training improves diastolic function in the isoproterenol-injected rats by ameliorating the ISO-associated deterioration in myocyte relaxation.

\section{CONCLUSION}

Our purpose was to gain a better understanding of the relation between ventricular remodeling and heart failure by assessing the adaptation of the heart through time to myocardial injury produced by ISO in the presence of both food restriction and physical activity. Exercise training and food restriction, when performed separately, produced a partial and, when performed together, a total preservation in the event of heart failure. The available data suggest that food restriction and exercise can improve the outcomes following heart failure. One of the mechanisms that seems to be responsible for early conservation in the event of heart failure is the restoration of the prooxidant-antioxidant balance. Also, we have demonstrated that this effect is related, at least in part, to enhancing angiogenesis via activation of HIF-1 $\alpha /$ VEGF mediated signaling pathway.

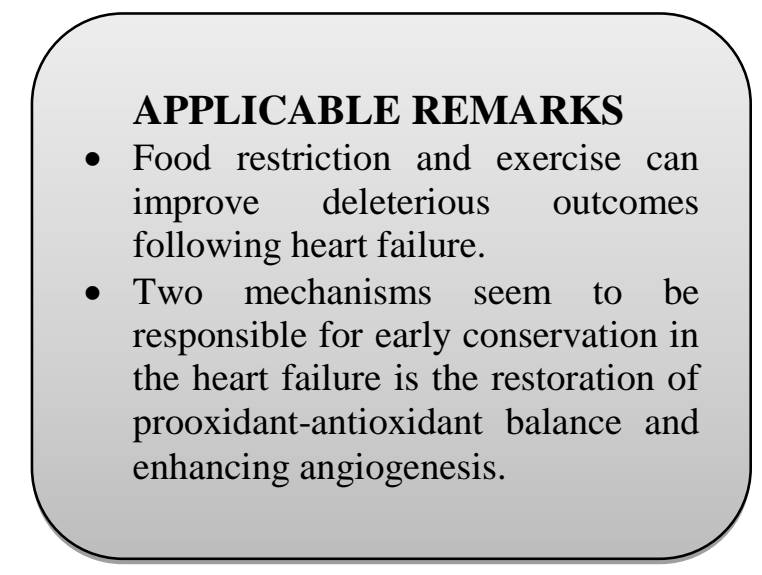

\section{REFERENCES}

1. Aronow WS. Epidemiology, pathophysiology, prognosis, and treatment of systolic and diastolic heart failure. Cardiology in review. 2006;14(3):108-24.

2. Alla F, Zannad F, Filippatos G. Epidemiology of acute heart failure syndromes. Heart failure reviews. 2007;12(2):91-5.

3. Murdoch CE, Zhang M, Cave AC, Shah AM. NADPH oxidase-dependent redox signalling in cardiac hypertrophy, remodelling and failure. Cardiovascular research. 2006;71(2):208-15.

4. Circu ML, Aw TY. Reactive oxygen species, cellular redox systems, and apoptosis. Free radical biology \& medicine. 2010;48(6):749-62.

5. Katare RG, Kakinuma Y, Arikawa M, Yamasaki F, Sato T. Chronic intermittent fasting improves the survival following large myocardial ischemia by activation of BDNF/VEGF/PI3K signaling pathway. Journal of molecular and cellular cardiology. 2009;46(3):405-12. 
6. Weiss EP, Racette SB, Villareal DT, Fontana L, Steger-May K, Schechtman KB, et al. Improvements in glucose tolerance and insulin action induced by increasing energy expenditure or decreasing energy intake: a randomized controlled trial. The American journal of clinical nutrition. 2006;84(5):1033-42.

7. Fontana L, Meyer TE, Klein S, Holloszy JO. Long-term calorie restriction is highly effective in reducing the risk for atherosclerosis in humans. Proceedings of the National Academy of Sciences of the United States of America. 2004;101(17):6659-63.

8. Ahmet I, Wan R, Mattson MP, Lakatta EG, Talan M. Cardioprotection by intermittent fasting in rats. Circulation. 2005;112(20):3115-21.

9. Spaulding CC, Walford RL, Effros RB. Calorie restriction inhibits the age-related dysregulation of the cytokines TNF-alpha and IL-6 in C3B10RF1 mice. Mechanisms of ageing and development. 1997;93(1-3):87-94.

10. Heil M, Schaper W. Influence of mechanical, cellular, and molecular factors on collateral artery growth (arteriogenesis). Circulation research. 2004;95(5):449-58.

11. Iglarz M, Silvestre JS, Duriez M, Henrion D, Levy BI. Chronic blockade of endothelin receptors improves ischemia-induced angiogenesis in rat hindlimbs through activation of vascular endothelial growth factor-no pathway. Arteriosclerosis, thrombosis, and vascular biology. 2001;21(10):1598-603.

12. Isner JM, Losordo DW. Therapeutic angiogenesis for heart failure. Nature medicine. 1999;5(5):491-2.

13. Gaesser GA. Exercise for prevention and treatment of cardiovascular disease, type 2 diabetes, and metabolic syndrome. Current diabetes reports. 2007;7(1):14-9.

14. Gaesser GA, Angadi SS, Sawyer BJ. Exercise and diet, independent of weight loss, improve cardiometabolic risk profile in overweight and obese individuals. The Physician and sportsmedicine. 2011;39(2):87-97.

15. Mercken EM, Carboneau BA, Krzysik-Walker SM, de Cabo R. Of mice and men: the benefits of caloric restriction, exercise, and mimetics. Ageing research reviews. 2012;11(3):390-8.

16. Shamsaei N, Khaksari M, Erfani S, Rajabi H, Aboutaleb N. Exercise preconditioning exhibits neuroprotective effects on hippocampal CA1 neuronal damage after cerebral ischemia. Neural regeneration research. 2015;10(8):1245-50.

17. Zaitone SA, Abo-Gresha NM. Rosuvastatin promotes angiogenesis and reverses isoproterenol-induced acute myocardial infarction in rats: role of iNOS and VEGF. European journal of pharmacology. 2012;691(1-3):13442 .

18. Schmittgen TD, Livak KJ. Analyzing real-time PCR data by the comparative $\mathrm{C}(\mathrm{T})$ method. Nature protocols. 2008;3(6):1101-8.

19. Afanas'ev I. ROS and RNS signaling in heart disorders: could antioxidant treatment be successful? Oxid Med Cell Longev. 2011;2011:293769.

20. Lopez-Lluch G, Hunt N, Jones B, Zhu M, Jamieson H, Hilmer S, et al. Calorie restriction induces mitochondrial biogenesis and bioenergetic efficiency. Proceedings of the National Academy of Sciences of the United States of America. 2006;103(6):1768-73.

21. Murry CE, Jennings RB, Reimer KA. Preconditioning with ischemia: a delay of lethal cell injury in ischemic myocardium. Circulation. 1986;74(5):1124-36.

22. Rajesh KG, Sasaguri S, Zhitian Z, Suzuki R, Asakai R, Maeda H. Second window of ischemic preconditioning regulates mitochondrial permeability transition pore by enhancing Bcl-2 expression. Cardiovascular research. 2003;59(2):297-307.

23. Mattson MP, Wan R. Beneficial effects of intermittent fasting and caloric restriction on the cardiovascular and cerebrovascular systems. J Nutr Biochem. 2005;16(3):129-37.

24. Seo AY, Hofer T, Sung B, Judge S, Chung HY, Leeuwenburgh C. Hepatic oxidative stress during aging: effects of 8\% long-term calorie restriction and lifelong exercise. Antioxidants \& redox signaling. 2006;8(3-4):529-38.

25. Wohlgemuth SE, Seo AY, Marzetti E, Lees HA, Leeuwenburgh C. Skeletal muscle autophagy and apoptosis during aging: effects of calorie restriction and life-long exercise. Experimental gerontology. 2010;45(2):138-48.

26. Takimoto E, Kass DA. Role of oxidative stress in cardiac hypertrophy and remodeling. Hypertension. 2007;49(2):241-8.

27. Golbidi S, Laher I. Molecular mechanisms in exercise-induced cardioprotection. Cardiology research and practice. 2011;2011:972807.

28. Tsutsui H, Kinugawa S, Matsushima S. Mitochondrial oxidative stress and dysfunction in myocardial remodelling. Cardiovascular research. 2009;81(3):449-56.

29. Meyer TE, Kovacs SJ, Ehsani AA, Klein S, Holloszy JO, Fontana L. Long-term caloric restriction ameliorates the decline in diastolic function in humans. Journal of the American College of Cardiology. 2006;47(2):398-402.

30. White FC, Bloor CM, McKirnan MD, Carroll SM. Exercise training in swine promotes growth of arteriolar bed and capillary angiogenesis in heart. Journal of applied physiology (Bethesda, Md : 1985). 1998;85(3):1160-8. 